BROOKHAVEN NATIONAL LABORATORY

Associated Universities, Inc.

Upton, New York

ACCELERATOR DE PARTMENT

Informal Report

PROPOSED REFINEMENTS TO RF BEAM GAPTURE PROCESS

AT INJECTION IN THE AGS

\title{
J.G. Cottingham
}

July 2, 1973

\begin{abstract}
The shortcomings of the present "Off Frequency" beam capture process are outlined and a system suggested to correct these. Frequency and $r$ amplitude jumps are generated electronivally to suvold transfer to pick-up electrode signals until later in the accelerating process when these aignals are clear or well-formed.
\end{abstract}

\section{N O T I C E}

This report was prepared as an account of work sponsored by the United States Government. Neither the United States nor the United States Atomic Energy Commission; nor any of their employees, nor any of their contractors, subcontractors, or their employees, makes any warranty, express or implied, or assumes any legal liability or responsibility for the accuracy, completeness or usefulness of any information, apparatus, product or process disclosed, or represents that its use would not infringe privately owned rights. 
The rf beam capture process used in routine operation of the AGS is the so-called "Off Frequency" variation of the "boot strap" capture process." 1-4 In this process, the beam is bunched by a starting oscillator whose frequency is different from that of the ideal particle. When the beam bunch is formed the frequency source is switched from the starting oscillator to a signal derived from the beam itself creating a frequency jump.

When all parameters of this capture system have been adjusted for best beam intensity, the following observations can be made:

1. Significant beam is lost during the first few milliseconds of time following transfer of control to the beam signal.

2. After the losses described in item 1 above, the populated phase region is smaller than the phase space available in the rf bucket.

3. The beam losses in item 1 above occur in a systematic and reproducible manner.

4. A.t the time of transfer to beam control the beam bunch is not yet well formed. Protons outside the rf accelerating bucket are still circulating in the AGS and influencing the pick-up electrode signal used for this control purpose.

5. There is a possibility that the controlling beam aignal is being altered by ion collection on the pick-up electrodes. The radial position information derived from these gignals are questionable. For this reason, it is common practice to blank the radial position correction signal for a short time.

6. Operations with high beam intensity $\left(\sim 10^{13}\right.$ particles) yields capture efficiencies near $50 \%$ which is disappointingly small and requires $2 \times 10^{13}$ particles circulating prior to capture.

These observations have lead to a reexamination of the capture process.

Simple adiabatic capture does not look promising because of the low rf accelerating voltage required, a few hundred vol ts per gap. The voltage induced by a beam of high intensity on the accelerating gap is of this magnitude, but 90 electrical degrees out of phase. The electronic problems associated with removing this induced voltage and generating the desired voltage are not easy to solve. 
The main difficulty with the "Off Frequency" capture process is the requirement to make the frequency jump before the beam bunch is well formed. Beam control itself is not required until later in the acceleration cycle near phase transition.

If the frequency jump required for capture were manufactured electronically, the transfer to beam control could be delayed until the beam is satisfactorily formed. The simplified block diagram shown in Fig. 1 accomplishes this. The variable frequency oscillator is programmed to generate the frequency of the central particle. This signal is phase advance prior to injection by a sequential array of phase locked oscillators used as phase shifters. During the bunching process this phase advance is linearly reduced to zero, which is equivalent to a frequency reduction. When the phase program reaches zero phase shift the Iinear fall stops producing no further phase shift. This is equivalent to a frequency jump to the frequency of the programmed variable oscillator automatically locking phase at the point of the frequency jump. Phase lock is required because the resonant if accelerating cavities, $Q=30$, will not respond to a step phase shift.

The electrical rf accelerating bucket formed after the frequency jump must be positioned on the beam populations so as to contain the maximum quantity of charge. Prior to the frequency jump the position of maximum beam intensity is not locked in phase to any part of the rf accelerating voltage. The jump must occur when the bunched beam passes through the desired phase point. This requirement quantizes the points in time where the frequency jump may occur for a given initial frequency offset. Figure 2 illustrates this relationship and is obtained from a computer model using typical parameters of the AGS. Plottrd here is the phase angle between the bunched beam and the $\mathrm{rf}$ voltage waveform as a function of time measured in revolutions. The four curves represent four different frequency offsets labeled as a fraction of the center frequency or as a fractional energy displacement of the electrical bucket from the central energy of the beam. These two fractions are related by

$$
\frac{\Delta f}{f}=-\frac{\eta}{\beta^{2}} \frac{d E}{E} \quad \text { where } \eta=\frac{1}{\nu^{2}}-\frac{E_{0}^{2}}{\left(E_{0}+T\right)^{2}}
$$


for the AGS at injection

$\nu=8.8, \mathrm{~T}=200 \mathrm{MeV}, \eta=-0.6665, \beta=0.5662$.

The beam bunch center is obtained by resolving the beam bunch shape into its Fourier components and defining the center to be the positive maximum of the first Fourier term. The relationship shown in Fig. 2 is produced by $180 \mathrm{kV}$ total crest voltage on the accelerating gaps. The variations of the phase plot from a straight 1 ine is produced by beam bunch shape fluctuations. In each case the approximately linear $r$ ise in phase is cerminated by a smearing of the general beam bunch shape after which the population density is dominated by the charge being carried along near the unstable fixed point.

In the coordinate system used, the stable phase point is $170^{\circ}$. When the beam bunch center passes through this angle, a frequency jump of proper magnitude will capture the maximum quantity of beam without requiring a forbidden phase shift in the accelerating voltage.

The curves of Fig. 2 cross the $170^{\circ}$ Iine 10 times. Again with the aid of the computer the shape in phase space of the beam populated region was examined at these 10 points. Eigure 3 shows this phase space shape for one of these points. The parameters used to generate the shape shown in Fig. 3 are; rf crest voltage $=180 \mathrm{kV}$, fractional frequency offset $=0.0062$, initial energy spread/mean beam energy $= \pm 0.000351$, number of revolutions of rf prior to frequency jump $=17$. The point chosen for illustration in Fig. 3 may not be the optimum, other points give similar shapes. But all of the points that show good bunching in phase space have characteristic shapes similar to that shown. The optimum operating point must be determined experimentally.

The beam populated region shown in Fig. 3 fits easily into the electrical bucket formed by a $50 \mathrm{kV}$ accelerating potential. This is a smaller potential than the $180 \mathrm{kV}$ used to form the bunch. The best bunch shape formed by $60 \mathrm{kV}$ is shown in Fig. 4. Clearly, this beam bunch is not of the same quality as that shown in Fig. 3, which gives rise to the desirability of reducing the rf potential coincident with the frequency jump. While $180 \mathrm{kV}$ is necessary to properly form the beam bunch, the electrical bucket produced by this potential is unnecessarily large and, if employed, will sweep particles into regions of high momentum. This consumes radial 
aperture which reduces the radial aperture available for betatron motion and result in beam loss.

Referring again to Fig. 3, it is clear that the center of charge is offset relative to center of the $60 \mathrm{kV}$ electrical bucket. This center of charge will revolve about the electrical center. The resulting phase motion is plotted in Fig. 5. Mixing of the populated region will ultimately damp this phase motion, but it is clear that many revolutions ( 1000) are required.

Figure 6 shows the phase space shape of the populated region after 80 revolutions which has carried the center of charge near its first phase minimum. The electrical bucket, represented by the solid line on Fig. 6, is the original $60 \mathrm{kV}$ accelerating bucket shown in Fig, 3 and the one used to compuie the motion of the populated region. The dashed line is an electrical bucket of the sane size but displaced in phase an amount equal to the phase motion of the center of charge. This represents the bucket that would result if the accelerating voltage were phase locked to the beam aignal. Clearly, beam that is contained in the undiaplaced bucket 18 lost, if the bucket were displaced. The beam populated area between the two buckets shown is approximately $4 \%$ of the total.

Also some particles inside the bucket near the periphery find themselves closer to the new periphery and may be lost from the bucket later as the bucket moves to the opposite extreme. A continuous shaving of the outer edges of the populated region results. This process explains the failure of the present beam controlled phase lock system to populate the full electrical bucket. The proposed revised systrm overcomes this process by delaying transfer to beam controlled phase lock until after the phase motion of the populated region has fully damped which is 5 to 10 millisecond after injection.

The total phase shift required to satisfy the parameters used in the illustrated example, Figs, 3,5 and 6, is $475^{\circ}$. However, this is not the maximum phase shift required to reach other possible frequency jump points. A total of $1650^{\circ}$ of available phase shift will allow the entire region illustrated in Fig. 2 to be explored, therefore 14 phase shifters each capable of $120^{\circ}$ will. be required. The variable frequency oscillator wich generates the scarting frequency should be programmed to match the correct center frequency for 10 milliseconds after injection. This will require a small correction proportional to time squared. 


\section{$\underline{\text { References }}$}

1. M. Plotkin, IRE Int. Conv. Record, Vol. 8, Part 9, P. 19-27 (1960).

2. W. Schne11, "Remark on the Phase Lock System of the CERN Proton Synchrotron," CERN Symp. 1959, 485-90.

3. K. Johnson, C. Schmelzer, Proc. CERN Symp. on High Energy Accelerators: p. 395 (1956).

4. W. Schnell, "Equivalent Circuit Anaiysis of Phase Lock Beam Control System," CERN 68-27, ISR Div. Rept. (1968).

Distr.: B2 


\section{Figure Captions}

Fig. 1 Block diagram for generating frequency jump with required phase lock.

Fig. 2 RF voltage - beam phase, degrees vs time measure in revolutions for various initial frequency offsets. $V=8.8, T=200 \mathrm{MeV}$ protons, rf voltage $=180 \mathrm{kV}$.

Fig. 3 Beam populated region - $60 \mathrm{kV}$ electrical accelerating bucket initial $\Delta E / E= \pm 0.003351,180 \mathrm{kV}, \Delta \mathrm{F} / \mathrm{F}=0.0062$, revolutions $=17$. Stable phase $=170^{\circ}$.

Fig. 4 Beam populated region - $60 \mathrm{kV}$ electrical accelerating bucket initial $\Delta \mathrm{E} / \mathrm{E}= \pm 0.000351,60 \mathrm{kV}, \Delta \mathrm{F} / \mathrm{F}=0.0036$, revilutions $=32$. Stabie phase $=170^{\circ}$.

Fig. 5 Phase motion of the center of the charged population vs revolutions. (For parameters see Fig. 3.)

Fig. 6 Beam populated region after 80 revolutions. (For parameters see Fig. 3.) 


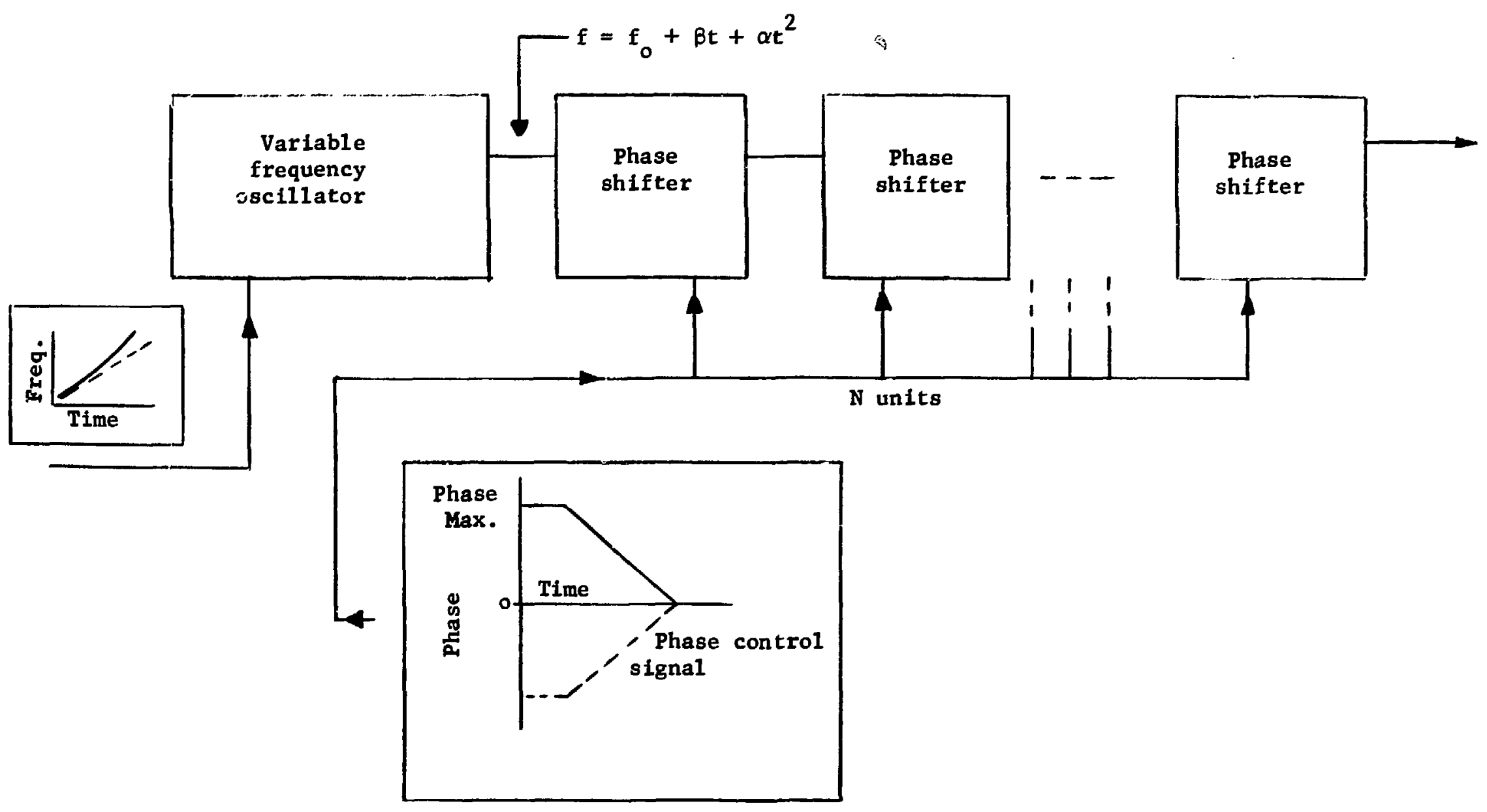

Fig. 1 


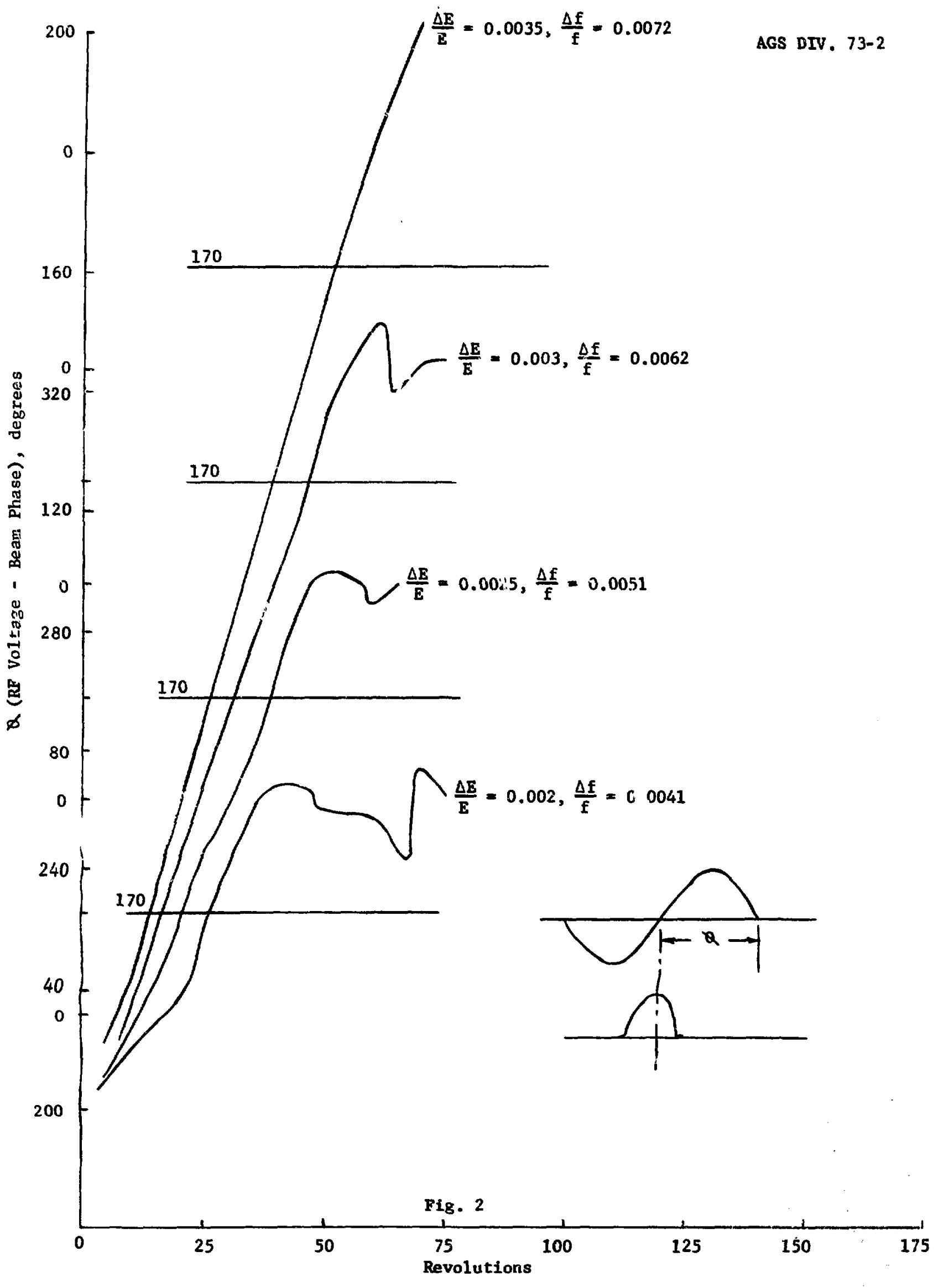


AGS DIV. 73-2

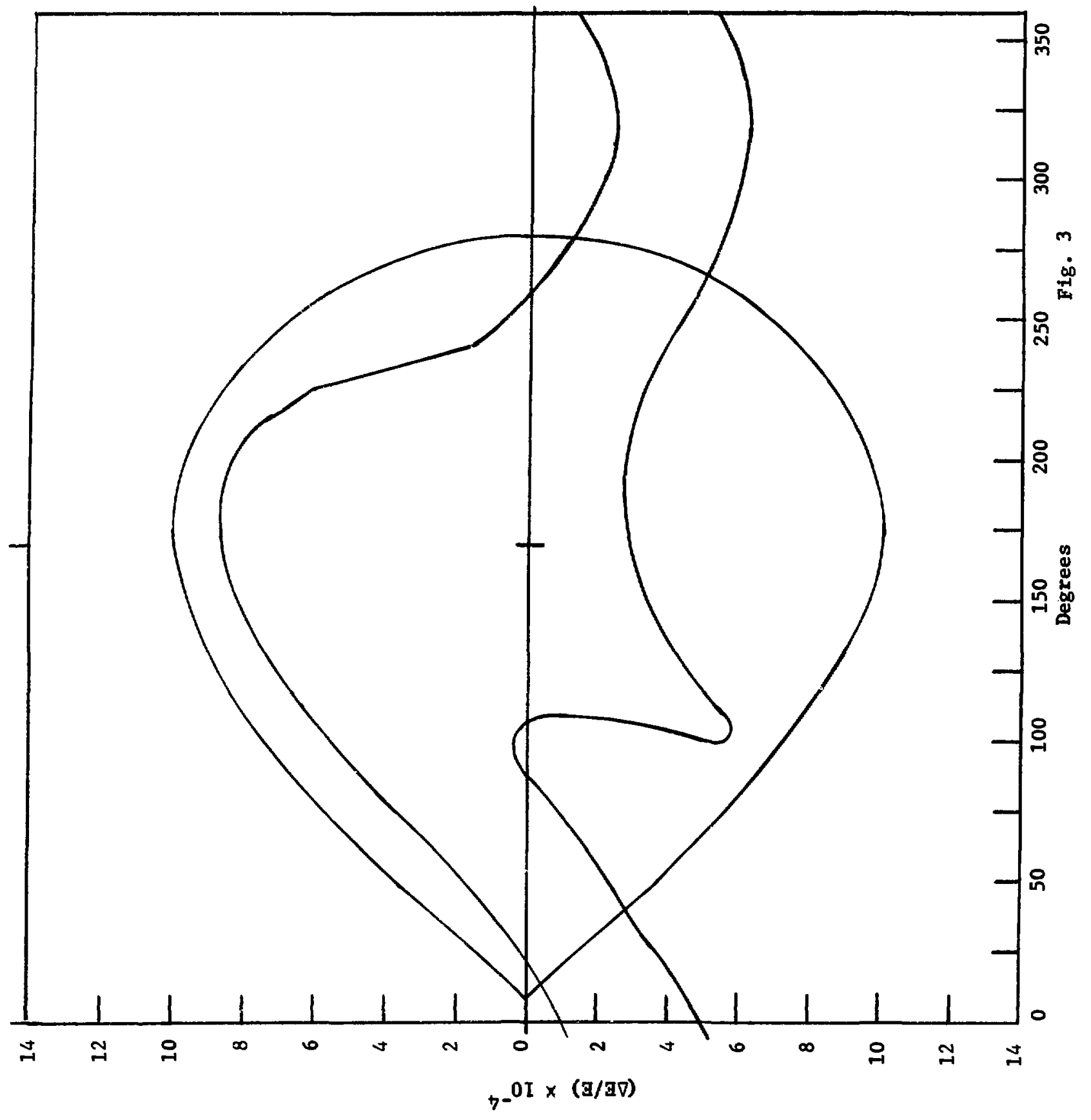




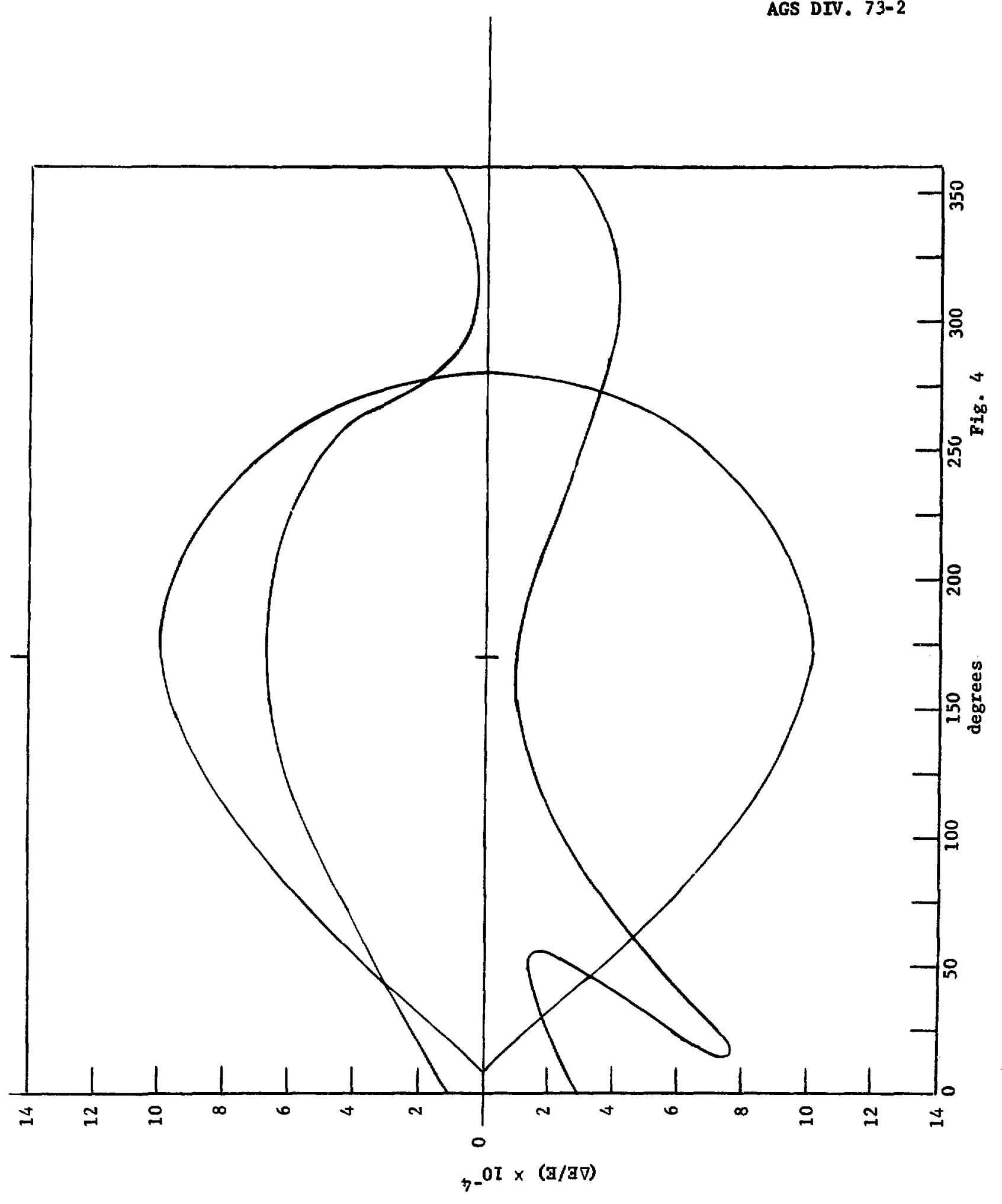




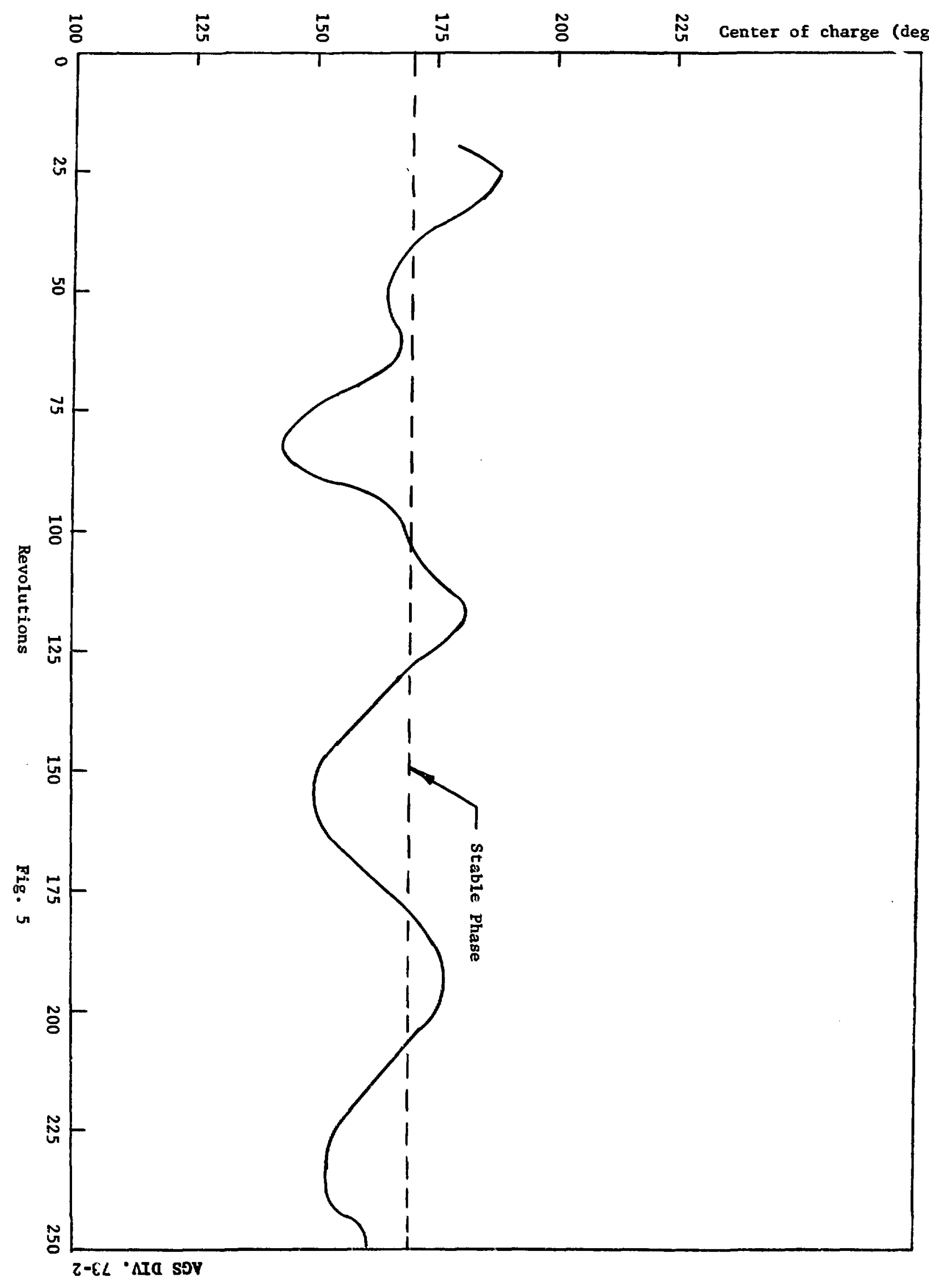


AGS DIV. 73-2

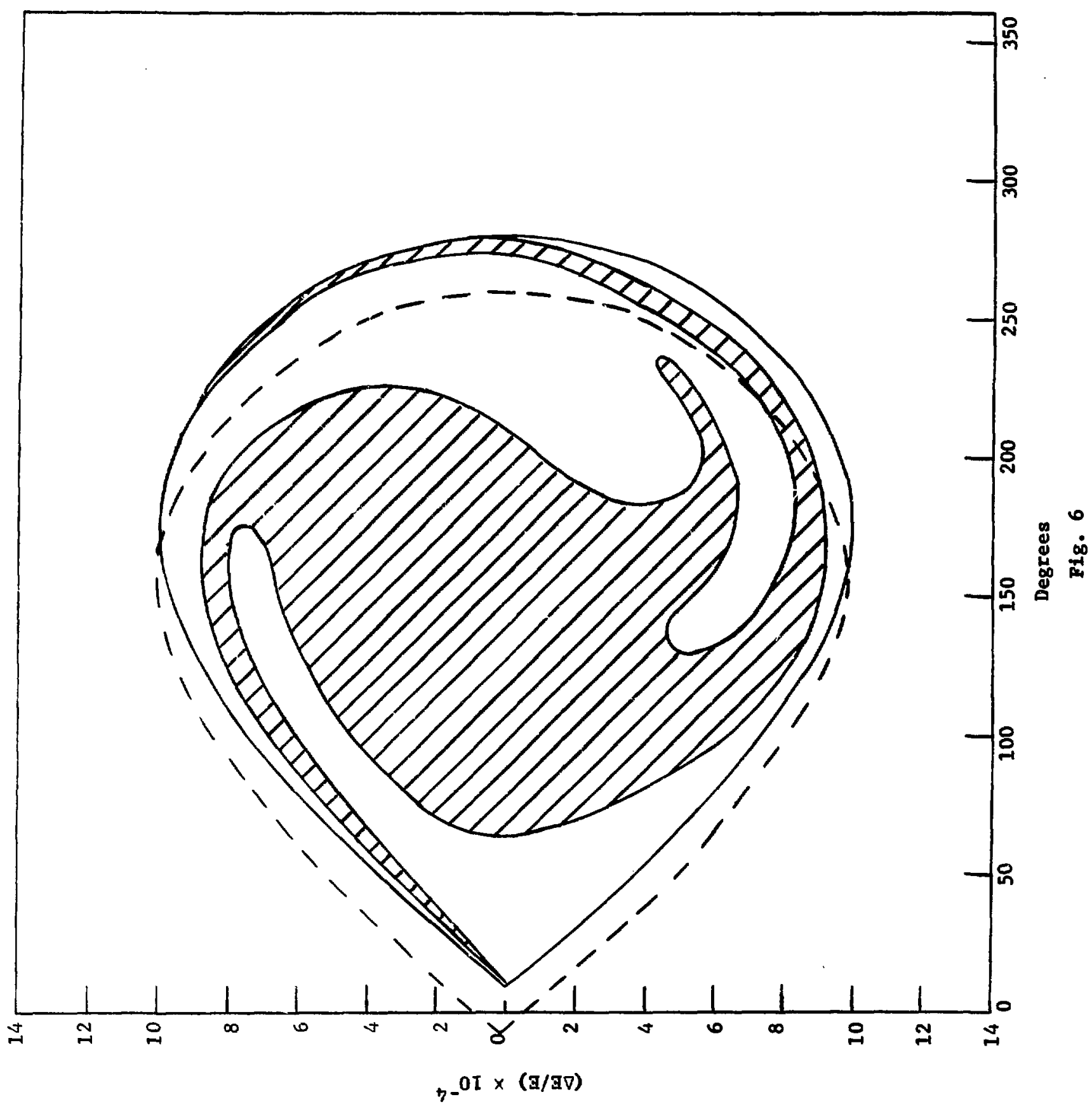

